\section{P629 GONORRHEA ACQUISITION AFTER INFECTION WITH THE US NMNG URETHRITIS CLADE: A PROSPECTIVE, CHART- BASED STUDY}

${ }^{1}$ Abigail Norris Turner*, ${ }^{2}$ Alexandria Carter, ${ }^{3}$ Morgan Brown, ${ }^{4}$ Yih-Ling Tzeng, ${ }^{4}$ David Stephens, ${ }^{2}$ Brandon Snyder, ${ }^{5}$ Devlin Prince, ${ }^{2}$ Jose Bazan. ${ }^{1}$ Ohio State University, Internal Medicine, Infectious Diseases, Columbus, USA; ${ }^{2}$ Ohio State University, College of Medicine, Internal Medicine, Infectious Diseases, Columbus, USA; ${ }^{3}$ Ohio State University, College of Public Health, Division of Epidemiology, Columbus, USA; ${ }^{4}$ Emory University, School of Medicine, Department of Medicine, Infectious Diseases, Atlanta, USA; ${ }^{5}$ Columbus Public Health, Columbus, USA

\subsection{6/sextrans-2019-sti.697}

Background Recent research suggests that Neisseria meningitidis $(\mathrm{Nm})$ OMV serogroup B vaccination protects against gonorrhea (caused by Neisseria gonorrhoeae, Ng). Since 2015, we have monitored a large cluster of urethritis cases caused by a uropathogenic, non-groupable $\mathrm{Nm}$ clade (US NmNG urethritis clade). The US NmNG urethritis clade encodes for MenB-4C vaccine antigens (FHbp, NhbA, NadA), but whether natural infection reduces subsequent risk of urethral gonorrhea is unknown.

Methods We constructed a dataset combining surveillance and medical record data from men diagnosed with US NmNG clade urethritis $(n=128)$ in a local STD clinic. We used timeto-event analyses of clinic visits between 1/2015 and 4/2018 to examine prospective urethral gonorrhea risk. As gonorrhea is a common event in STD patients, we compared subsequent gonorrhea acquisition for men with US NmNG clade urethritis at baseline to men with $\mathrm{Ng}$ urethritis $(\mathrm{n}=255)$, chlamydial urethritis $(n=253)$, or no infection $(n=257)$ at baseline.

Results Participants were primarily Black (65\%) and heterosexual (82\%), with a median age of 28 years. At baseline, 13\% had prior gonorrhea history. Only one participant had prior MenB vaccination. Half (49\%) of men returned for STD screening at least once during the follow-up period. Men with US NmNG clade urethritis at baseline had similar gonorrhea risk as men with $\mathrm{Ng}$ at baseline (HR: 1.03, 95\% CI: 0.601.76). Results were not meaningfully different when assessing extragenital gonococcal infections, or after adjustment for time since baseline, age, race, sexual orientation, prior gonorrhea infection, and sexual behavior (number of partners, condom use, and oral sex). In contrast, those with US NmNG clade urethritis had increased gonorrhea incidence compared to men with chlamydial urethritis (HR: 2.02, 95\% CI: 1.11-3.69) and men with no infection at baseline (HR: 3.84, 95\% CI: $1.87-$ 7.91).

Conclusion Natural infection with US NmNG urethritis clade does not appear to protect men against subsequent acquisition of gonorrhea.

Disclosure No significant relationships.

\section{P630 ESTIMATING THE POTENTIAL IMPACT OF REALISTIC VACCINATION STRATEGIES AGAINST ANTIBIOTIC RESISTANT NEISSERIA GONORRHOEAE}

'Lilith Whittles*, 'Peter White, ${ }^{2}$ Xavier Didelot. ${ }^{1}$ Imperial College School of Public Health, MRC Centre for Global Infectious Disease Analysis and NIHR Health Protection Research Unit in Modelling Methodology, Department of Infectious Disease Epidemiology, London, UK; ${ }^{2}$ University of Warwick, School of Life Sciences and Department of Statistics, Coventry, $U K$

10.1136/sextrans-2019-sti.698
Background Gonorrhoea infection is increasing and becoming harder to treat. In England, incidence among men who have sex with men (MSM) has increased eight-fold since 2008, reaching $\geq 21,000$ cases in 2017 . This epidemic, coupled with the growing threat of potentially untreatable antibiotic-resistant infection, has renewed interest in a gonococcal vaccine. Previous vaccine development attempts have failed; however, observational evidence suggesting the MeNZB meningococcal $\mathrm{B}$ vaccine is partially protective against gonorrhoea, with $31 \%$ effectiveness but uncertain duration, indicates it may be possible to develop a suitable vaccine.

Methods We fitted a stochastic transmission-dynamic model, incorporating asymptomatic and symptomatic infection and heterogeneous sexual behaviour, to gonorrhoea incidence in MSM in England over 2008-17 using particle Markov Chain Monte Carlo methods. Bayesian forecasting, considering realistic vaccination strategies under different scenarios of antibiotic resistance, determined how vaccine effectiveness and duration of protection affect population-level impact, and examined feasibility of achieving WHO's target of reducing gonorrhoea incidence by $90 \%$ between 2016 and 2030 .

Results Even a partially-effective vaccine could have a substantial impact if protection lasts long enough. In a worst-case scenario of untreatable gonorrhoea, vaccinating all MSM attending sexual health clinics with a $58 \%$ effective vaccine protecting for $\geq 12$ years (with boosters if required), or a $66 \%$ effective vaccine lasting $\geq 6$ years, reduces expected incidence below the WHO target. A vaccine conferring 30\% protection for 2-4 years reduces expected incidence in 2030 by $34 \%$ if gonorrhoea becomes untreatable, but if $\geq 80 \%$ of gonorrhoea cases are treatable then incidence is reduced by $95 \%$.

Conclusion Our statistically rigorous assessment shows that even a partially-effective vaccine, delivered through a practical targeting strategy, could have a substantial benefit in reducing gonorrhoea incidence in the context of an epidemic with rising antibiotic resistance. Our model can help design trials to measure vaccine effectiveness and duration of protection and assess cost-effectiveness of vaccination strategies.

Disclosure No significant relationships.

\section{P631 CASES OF GONORRHEA WITH MULTIPLE ANATOMIC SITES OF INFECTION IN CANADA, 2016 TO 2017}

${ }^{1}$ Pam Sawatzky*, ${ }^{2}$ Irene Martin, ${ }^{3}$ Vanessa Allen, ${ }^{4}$ Linda Hoang, ${ }^{5}$ Paul Van Caessele, ${ }^{6}$ Prenilla Naidu, ${ }^{7}$ Mike Mulvey. ${ }^{1}$ Public Health Agency of Canada, National Microbiology Laboratory, Winnipeg, Canada; ${ }^{2}$ National Microbiology Laboratory, Winnipeg, Canada; ${ }^{3}$ Public Health Ontario, Toronto, Canada; ${ }^{4}$ British Columbia Centres for Disease Control, Vancouver, Canada; ${ }^{5}$ Cadham Provincial Laboratory, Winnipeg, Canada; ${ }^{6}$ Provincial Laboratory for Public Health, Edmonton, Canada; ${ }^{7}$ Public Health Agency of Canada, Winnipeg, Canada

10.1136/sextrans-2019-sti.699

Background Neisseria gonorrhoeae, the causative agent of gonorrhea, is the second most commonly reported bacterial sexually transmitted infection in Canada and a global public health threat. Many cases of gonococcal infection include isolates from more than one anatomical infection site. This study compares the antimicrobial resistance (AMR) and molecular sequence type (ST) of multiple anatomical infection site isolates from individual cases.

Methods Isolates were collected by Canadian provincial laboratories and submitted to the National Microbiology Laboratory (NML) from 2016-2017. AMR profiles were determined using 